\title{
Sleep Disturbances in Children with Attention Deficit Hyperactivity Disorder
}

\author{
LOUISE MARGARET O'BRIEN, ANNA IVANENKO, VALERIE MCLAUGHLIN CRABTREE, \\ CHERYL ROBIN HOLBROOK, JENNIFER LEIGH BRUNER, CARRIE JOANN KLAUS, \\ DAVID GOZAL
}

Kosair Children's Hospital Research Institute, and Division of Pediatric Sleep Medicine, Department of Pediatrics, University of Louisville, Louisville, Kentucky 40204, U.S.A.

\section{ABSTRACT}

\begin{abstract}
The objective of the study was to compare polysomnographic patterns in two groups of preadolescent children with attention deficit hyperactivity disorder (ADHD) (a sleep clinic referral sample and a community sample) with control children. A prospective and observational study in the sleep clinic and the community was undertaken. Forty-seven ADHD children referred to the sleep clinic (ADHDcl), 53 ADHD children from a community survey (ADHDcom), and 49 control children underwent overnight polysomnography. Significant differences between the groups were observed for rapid-eye-movement (REM) sleep latency and percentage, and periodic limb movement index with associated arousals (PLMa). REM sleep latency was shorter in controls than ADHDcl and ADHDcom $(p<0.01)$ and REM\% was highest in controls and lowest in ADHDcl $(p<0.001)$. PLMa was higher in ADHDcl than the other groups $(p<0.001)$, but there were no differences in PLMa between ADHDcom and controls. ADHD children display significant alterations in their sleep patterns, and ADHDcl are more likely to have an elevated PLMa than ADHDcom. In addition, REM sleep is affected by ADHD. We postulate that ADHDcl may represent a subset of children with ADHD at high risk for hyperactivity during sleep. (Pediatr Res 54: 237-243, 2003)
\end{abstract}

\author{
Abbreviations \\ ADHD, attention/deficit hyperactivity disorder \\ ADHDcl, clinical sample of children with ADHD \\ ADHDcom, community sample of children with ADHD \\ AHI, apnea/hypopnea index \\ AI, apnea index \\ EDS, excessive daytime sleepiness \\ EOG, electrooculogram \\ EMG, electromyogram \\ PLMa, periodic limb movements with arousals \\ PLMD, periodic limb movement disorder \\ PLMI, periodic limb movement index \\ PLMS, periodic leg movements in sleep \\ PSG, polysomnography \\ REM, rapid eye movement \\ REM\%, duration of REM sleep as percentage of total sleep \\ time \\ SDB, sleep-disordered breathing \\ $\mathrm{SpO}_{2}$, oxyhemoglobin saturation \\ SWS, slow-wave sleep \\ TST, total sleep time
}

Parental reports of sleep disturbances are common in children with ADHD (1-8) and were previously included as one of the Diagnostic and Statistical Manual of Mental Disorders, Third Edition, diagnostic criteria for attention deficit disorder (9). The study of sleep problems in ADHD may be particularly important because disturbances in sleep, such as SDB (2, 10-14), narcolepsy (15), and PLMS (16-19), may present symptoms that resemble ADHD.

Received June 25, 2002; accepted February 3, 2003

Correspondence: David Gozal, Kosair Children's Hospital Research Institute, University of Louisville School of Medicine, 571 S. Preston St., Suite 321, Louisville, KY 40202, U.S.A.; e-mail: david.gozal@louisville.edu

Supported by National Institutes of Health Grant HL-65270, the Department of Education Grant H324E011001, and The Commonwealth of Kentucky Research Challenge Trust Fund.

DOI: 10.1203/01.PDR.0000072333.11711.9A
In a survey of sleep habits of 46 children with ADHD and 46 controls aged 5-10 y, Owens et al. (7) showed that children with ADHD scored significantly higher on all sleep subscales than controls, i.e. increased bedtime resistance, sleep-onset delay, sleep duration, sleep anxiety, and excessive daytime sleepiness. The children in this study also completed questionnaires, and those with ADHD also reported more sleep disturbance than controls. In a three-stage study by Kaplan et al. (1), the validity of clinical reports of increased sleep disturbance in ADHD children was evaluated. In both clinical and community settings, parents of ADHD children perceived far more sleep disturbances than controls. However, daily documentation to minimize reporting bias demonstrated that ADHD children did seem to differ from controls with regard to sleep duration, number of awakenings, and enuresis, but not with regard to sleep latency or total sleep time. 
In contrast to the findings obtained from subjective information, the few studies that have objectively quantified sleep characteristics in the ADHD population (20-23) have been unable to identify striking and reproducible differences between ADHD and control children. Busby et al. (20) performed overnight polysomnography in a small number of children with ADHD $(n=11)$ and controls $(n=11)$ and found that REM sleep latency was significantly increased in the ADHD group, a finding that contrasts with Kahn (24), who found a decreased REM sleep latency, and also with Ramos Platon et al. (23), who found no change. Sleep latency has been shown to be reduced in ADHD (23), not changed (20), and even increased (21), although the latter study only demonstrated an increased sleep latency in methylphenidate-treated children with ADHD and no difference in sleep latency when children were not medicated. Additionally, these studies of sleep architecture did not include any assessment of cardiorespiratory measurements.

Children with PLMS are reported to have hyperactive behavior (16) and PLMS may be common among hyperactive children $(17-19,25)$. In one study by Picchietti and Walters (18), 15 out of 16 children with frequent PLMS ( $>25$ per hour of sleep) had ADHD. Chervin and Archbold (26) further reported a dose-dependent association between hyperactivity, measured by the Conners' Parent Rating Scale, and PLMI (27), and indeed treatment of PLMD will often lead to substantial behavioral improvements (28).

It should be stressed, however, that the majority of the published studies on objective assessments of sleep in ADHD have based their findings on highly selective patient samples recruited from either psychiatric or neurologic clinics, for the most part have used small sample sizes, and either have not included controls or the control populations were inadequate.

In an effort to reconcile the above-mentioned findings and further delineate the nature of sleep-related disturbances in children with ADHD, we conducted parental surveys and overnight sleep studies in three different groups of preadolescent children, namely a group of ADHD children recruited from a sleep clinic referral sample, a group of ADHD children recruited from a community-based sample, and control children.

\section{METHODS}

Subjects. The clinical sample of children with ADHD was identified from a chart review of the pediatric population of children referred to Kosair Children's Hospital Sleep Medicine and Apnea Center during the years 2000-2002, and who underwent overnight PSG as part of their clinical assessment (ADHDcl). Consecutive children with ADHD diagnosed by pediatricians, psychiatrists, and/or psychologists were included. Children were excluded if they had a co-morbid psychiatric diagnosis because of the potential effect of other psychiatric diagnoses and medications on sleep. Information about sleep habits was routinely obtained from the parents during the initial clinic appointment and documented using a problem-based survey instrument (Appendix 1). The sleep questionnaire focused on the presence of sleep disturbances, e.g. daytime sleepiness, restless sleep, sleepwalking, nightmares, enuresis, witnessed apnea, and snoring.

The community sample (ADHDcom) was recruited from a survey of sleeping habits conducted among the parents of 5- to 7-y-old children attending Jefferson County Public Schools system using a previously validated questionnaire (Appendix 1) (29). Children were randomly selected as ADHDcom if they answered affirmatively to the question "Does your child have ADHD?" or "Is your child hyperactive?" Controls were randomly selected from those children with no medical conditions and no hyperactivity. Both ADHDcom and controls were invited for an overnight PSG assessment in the sleep laboratory. To objectively confirm or refute the presence of hyperactivity in the ADHDcom and control groups, all children were assessed using the ADHD subscale of the Conners' ADHD index (27). A score of at least 2 SD above the mean (a score $\geq 70$ ) on this subscale was used to classify the children as hyperactive. In addition, children from the community who had received a professional diagnosis of ADHD and who were taking stimulant medication were also included in the community group of hyperactive children. Those children who scored $<1$ SD above the mean on the ADHD index of the Conners' Parent Rating Scale comprised the control group. Children with neurodevelopmental disabilities and craniofacial abnormalities were excluded from the study. This study was approved by the University of Louisville Institutional Review Board. Parental informed consent and child assent, in the presence of a parent, were obtained.

PSG assessment. A standard overnight multichannel PSG evaluation was performed at the Sleep Medicine Center of Kosair Children's Hospital. Children were studied for up to $12 \mathrm{~h}$ in a quiet, darkened room with an ambient temperature of $24^{\circ} \mathrm{C}$ in the company of one of their parents. All studies were terminated when the children woke up for the day or at approximately $0700 \mathrm{~h}$ if they were still sleeping whether or not the studies were performed on a school night or a weekend. No drugs were used to induce sleep. The following parameters were measured: chest and abdominal wall movement by respiratory impedance or inductance plethysmography; heart rate by ECG; air flow monitored with a sidestream end-tidal capnograph, which also provided breath-by-breath assessment of end-tidal carbon dioxide levels ( Peтco $_{2}$; Pryon SC-300, Menomonee Falls, WI, U.S.A.), and/or a thermistor. $\mathrm{SpO}_{2}$ was assessed by pulse oximetry (Nellcor N 100; Nellcor Inc., Hayward, CA, U.S.A.), with simultaneous recording of the pulse waveform. The bilateral EOG, eight channels of EEG, chin and anterior tibial EMG, and analog output from a body position sensor (Braebon Medical Corporation, Ogdensburg, NY, U.S.A.) were also monitored. All measures were digitized using a commercially available PSG system (Stellate Systems, Montreal, Quebec, Canada). Tracheal sound was monitored with a microphone sensor (Sleepmate, Midlothian, VA, U.S.A.) and a digital time-synchronized video recording was performed.

Sleep variables. Sleep architecture was assessed by standard techniques (30). The AI was defined as the number of apneas per hour of TST. Hypopneas were defined as a decrease in nasal flow of $\geq 50 \%$ with a corresponding decrease in $\mathrm{SpO}_{2}$ of 
$\geq 4 \%$ and/or an arousal. The AHI was defined as the number of apneas and hypopneas per hour of TST and the AI was defined as the number of apneas per hour of TST. The mean $\mathrm{SpO}_{2}$, as measured by pulse oximetry, together with $\mathrm{SpO}_{2}$ nadir, were determined. The mean and peak $\mathrm{PETCO}_{2}$ were determined. Arousals were defined as recommended by the American Sleep Disorders Association Task Force report (31) and include respiratory-related (occurring immediately after an apnea, hypopnea, or snore), technician-induced, and spontaneous arousals. Arousals were expressed as the total number of arousals per hour of sleep time (arousal index). Central, obstructive, and mixed apneic events were counted. Obstructive apnea was defined as the absence of airflow with continued chest wall and abdominal movement for a duration of at least two breaths (32, 33). PLM during sleep were scored if there were at least four movements of $0.5-$ to 5 -s duration, and between 5 and $90 \mathrm{~s}$ apart. A PLMI of $>5$ per hour of sleep is generally considered to be rare in normal children (34). PLM associated with arousals (PLMa) were also scored if the arousal occurred $<1 \mathrm{~s}$ after the PLM. A PLMa index of $\geq 1$ was considered significant.

Data analysis. Data are presented as means \pm SD unless otherwise indicated. For questionnaire-derived responses, comparisons were made with $\chi^{2}$ analyses (dichotomous outcomes). ANOVA was used for comparisons of PSG measures between the study groups, with $p$ values adjusted for unequal variances using the Dunnett's T3 statistic when appropriate. Stepwise logistic regression was performed to evaluate the relationship between the sleep measures within the study groups. All $p$ values reported are two-sided with statistical significance set at $<0.05$.

\section{RESULTS}

Parental surveys. Information about sleep habits and problems was routinely obtained from the parents during the clinic appointment in ADHDcl subjects. The majority of children (79\%) presented with a chief complaint of suspected SDB. A total of 47 children were eligible for inclusion in the ADHDcl group (35 male). Subjective sleep information was similarly obtained from 53 ADHDcom (31 male) and 49 controls (22 male). Table 1 illustrates the differences in subjective sleep reports between the three groups. A further 62 children were

Table 1. Frequency of parentally reported sleep problems among $A D H D$ and control children

\begin{tabular}{lccc}
\hline \multicolumn{1}{c}{ Variable } & $\begin{array}{c}\text { ADHDcl, } \\
n=47(\%)\end{array}$ & $\begin{array}{c}\text { ADHDcom, } \\
n=53(\%)\end{array}$ & $\begin{array}{c}\text { Control, } \\
n=49(\%)\end{array}$ \\
\hline Sleeps alone & 79 & 62 & 63 \\
Difficulty initiating sleep & $52^{* *}$ & $47 * *$ & 14 \\
Restless sleep & $91 * *$ & $82^{* *}$ & 37 \\
Nightmares & $62^{* * \S}$ & $23^{*}$ & 6 \\
Sleep walking & $31^{* *}$ & 17 & 6 \\
Enuresis & $51^{* * \mathbb{I}}$ & 25 & 12 \\
Stops breathing & $40^{* * \S}$ & 9 & 2 \\
EDS & $53^{* *}$ & $49 * *$ & 12 \\
\hline
\end{tabular}

$* p \leq 0.05$ vs controls.

$* * p \leq 0.01$ vs controls.

II $p \leq 0.05$ ADHDcl $v s$ ADHDcom.

$\S p \leq 0.001$ ADHDcl vs ADHDcom. recruited on the basis of parental report of ADHD but on further assessment were found not to fulfill criteria for inclusion in the ADHDcom group. There were no differences in any of the subjective complaints for the 62 excluded children compared with the children who were included $(n=53)$, although there were more males in the nonstudied group $(p<$ $0.03)$.

Controls were significantly different from the ADHDcl $(p<$ 0.01) for all sleep questionnaire variables except sleeping alone, where there was no difference between the groups. The control group also demonstrated fewer difficulties with initiating sleep $(p<0.001)$, restless sleep $(p<0.0001)$, nightmares $(p<0.05)$, and excessive sleepiness $(p=0.0002)$ than the ADHDcom group. Compared with ADHDcom, ADHDcl were significantly more likely to complain of nightmares $(p=$ $0.0002)$, enuresis $(p<0.02)$, and witnessed apnea $(p<0.01)$.

Polysomnographic findings. Table 2 shows the demographic information for the three groups of children and Table 3 shows the results of the PSG. The ADHDcl group was significantly older than both the ADHDcom and control groups $(p<0.01)$ and had a larger proportion of males than the control group $(p<0.01)$.

ANOVA revealed statistically significant differences between the groups for REM sleep latency and REM\%, total percentage of SWS (SWS\%; as a percentage of total sleep time), spontaneous arousal index, and periodic leg movement index associated with arousals (PLMa). REM sleep latency was shorter in controls than in ADHDcl $(p=0.003)$ and $\operatorname{ADHDcom}(p=0.004)$ and REM $\%$ was significantly higher in controls than both ADHDcl and ADHDcom $(p<0.001)$. The ADHDcom group had more SWS than the ADHDcom group ( $p$ $<0.04)$. ADHDcl showed significantly lower spontaneous arousals $(p<0.001)$ than both ADHDcom and controls. Fifteen (32\%) ADHDcl children had a PLMI $\geq 5$, compared with eight (15\%) ADHDcom and nine (18\%) control children $(p=\mathrm{NS})$. Mean PLMa was also higher in ADHDcl than in ADHDcom and controls $(p<0.001)$, but there were no differences in PLMa between ADHDcom and controls. In addition, 38\% of ADHDcl had a PLMa $\geq 1$ compared with only $4 \%$ of ADHDcom and none of the controls $(p<0.001$ ADHDcl versus other groups).

Stepwise regression analysis revealed that REM\% and PLMa together account for $44 \%$ of the dependent variable (ADHD or control) (Nagelkerke $R^{2}$ ).

Table 2. Demographic characteristics of ADHDcl, ADHDcom, and control children undergoing an overnight polysomnographic evaluation

\begin{tabular}{lccc}
\hline & $\begin{array}{c}\text { ADHDcl, } \\
n=47\end{array}$ & $\begin{array}{c}\text { ADHDcom, } \\
n=53\end{array}$ & $\begin{array}{c}\text { Control, } \\
n=49\end{array}$ \\
\hline Age (y) & $\begin{array}{c}8.0 \pm \\
1.6 * * \S\end{array}$ & $6.6 \pm 0.4$ & $6.7 \pm 0.4$ \\
Male gender & $35(74 \%)^{* *}$ & $31(58 \%)$ & $22(44 \%)$ \\
Stimulant medicated & $34(72 \%)^{* * \mathbb{I}}$ & $27(51 \%)^{* *}$ & 0
\end{tabular}

$* p<0.05$ for index $v s$ controls.

** $p<0.01$ for index $v s$ controls.

If $p<0.05$ for ADHDcl $v s$ ADHDcom.

$\S p<0.01$ for ADHDcl vs ADHDcom. 
Table 3. Polysomnographic characteristics of ADHDcl, ADHDcom, and control children

\begin{tabular}{|c|c|c|c|}
\hline Variable & $\begin{array}{l}\text { ADHDcl, } \\
n=47\end{array}$ & $\begin{array}{l}\text { ADHDcom, } \\
n=53\end{array}$ & $\begin{array}{l}\text { Control, } \\
n=49\end{array}$ \\
\hline Sleep efficiency $(\%)$ & $86.9 \pm 9.1$ & $89.4 \pm 7.7$ & $90.0 \pm 6.7$ \\
\hline Sleep latency (min) & $20.9 \pm 18.2$ & $32.4 \pm 32.8$ & $25.8 \pm 25.1$ \\
\hline REM sleep latency (min) & $161.1 \pm 74.7 * *$ & $159.0 \pm 71.2 * *$ & $121.3 \pm 47.3$ \\
\hline REM sleep $(\%)$ & $17.7 \pm 6.3^{* *} \S$ & $20.9 \pm 6.4 * *$ & $27.5 \pm 5.2$ \\
\hline SWS $(\%)$ & $24.5 \pm 5.6 \rrbracket$ & $21.9 \pm 7.2$ & $22.3 \pm 4.9$ \\
\hline $\begin{array}{l}\text { Spontaneous arousal } \\
\text { index }\end{array}$ & $5.2 \pm 2.3^{* *} \S$ & $9.3 \pm 4.3$ & $8.5 \pm 3.6$ \\
\hline Respiratory arousal index & $0.4 \pm 1.0$ & $1.3 \pm 2.8$ & $0.5 \pm 0.9$ \\
\hline PLM index & $2.8 \pm 3.5$ & $2.4 \pm 5.0$ & $2.4 \pm 3.9$ \\
\hline PLMa index & $1.1 \pm 1.3 * * \S$ & $0.1 \pm 0.4$ & $0.1 \pm 0.2$ \\
\hline AHI & $1.0 \pm 1.8$ & $1.4 \pm 1.9$ & $0.8 \pm 0.7$ \\
\hline AI & $0.8 \pm 0.9$ & $0.7 \pm 0.8$ & $0.7 \pm 0.5$ \\
\hline $\mathrm{SpO}_{2}$ nadir $(\%)$ & $92.5 \pm 6.4$ & $91.5 \pm 4.8$ & $93.0 \pm 3.3$ \\
\hline \multicolumn{4}{|l|}{$* p<0.05$ vs controls. } \\
\hline
\end{tabular}

\section{DISCUSSION}

This study illustrates that prepubertal children with ADHD display significant alterations in REM sleep patterns and, furthermore, children with ADHD referred to a sleep clinic are more likely to have an elevated PLMa compared with ADHD children from the general community. Our findings further suggest that REM\% and PLMa together account for nearly half of the variance observed between index and control groups.

The current study is, to our knowledge, the largest to date to compare subjective and objective reports of sleep problems in children with ADHD and controls and, furthermore, it is unique in that we had the opportunity to investigate a referred population as well as a community population of children with ADHD. Our subjective findings are in agreement with previous studies (1-8), and show that parents of ADHD children report significantly more problems with sleep, which include increased bedtime resistance $(7,8)$, difficulty initiating sleep $(1$, $5,7,8)$, nighttime awakenings $(1,7)$, sleep-related anxiety $(3$, 7), enuresis (5), and excessive daytime sleepiness (7). In fact, a comprehensive review of the literature (4) revealed that parents of children with ADHD were five times as likely to report that their children have sleep problems compared with parents of healthy children.

Despite such high frequency of sleep-related complaints, objective assessments of the sleep of children with ADHD have shown inconsistent findings (20-23). However, small sample sizes and inconsistent criteria make comparisons of these studies difficult. The disparity between subjective and objective findings is consistent with that found in the present study and reported by Corkum et al. (8), who compared 25 children with ADHD with 25 age-matched controls (mean age, 9 y) using parent- and child-reports of sleep quality and 7-d actigraphic recordings. Parents of children diagnosed with ADHD reported significantly more problems with sleep onset, morning awakenings, restless sleep, and bedtime resistance than children in the control group. However, when both actigraphy recordings and the children's report of their own sleep were analyzed, the only variable that was significantly different between the two groups was bedtime resistance. Corkum and colleagues hypothesized that the significant difficulties that parents of children with ADHD report with their children's sleep may be related to the difficult and often oppositional behaviors manifesting as bedtime resistance rather than represent primary sleep disorders in ADHD. In addition, the objective-subjective incongruences could also be related to differences introduced by retrospective and prospective collection of sleep measures.

Clinically, parents of children with ADHD complain of hyperactive and oppositional behaviors that occur throughout the day and into the evening. The extension of these behaviors to bedtime is the most likely explanation for the higher probability that parents of children with ADHD will report that their children have bedtime resistance and sleep-onset delay. Thus, children with ADHD may be displaying oppositional behaviors that prevent them from following rules and engaging in appropriate bedtime behaviors, and therefore reflect more sleep limit-setting issues rather than represent sleep disorders per se.

In contrast with other studies $(16-19,25,26)$ we did not find an increase in PLMI in our ADHD children. However, the clinic sample tended to show a greater proportion of children with PLMI $\geq 5$, although this did not reach statistical significance. Interestingly, significantly more of the ADHDcl group demonstrated arousals associated with the PLM. Sampling bias is the most likely explanation for these findings. Thus, children presenting to clinics are more likely to have more severe sleep problems than those children not in need of specialty care services. The samples of children with ADHD recruited by Picchietti and colleagues (17-19) appear similar to those children enrolled in the ADHDcl group of the present study inasmuch as they presented to a clinic setting for treatment of behavioral and sleep difficulties associated with their ADHD. We postulate that the children in our ADHDcom sample are most likely to reflect the vast majority of children with ADHD who are functioning without the assistance of specialized sleep, psychiatry, or neurology clinics. This further poses the question of whether those children attending the clinical setting are actually a subgroup of children with ADHD who are also more likely to have a nighttime disorder (i.e. PLMD). In other words, ADHD children referred to a tertiary specialty clinic may represent a subgroup of ADHD children who are at risk for PLMD, which in turn may exacerbate daytime behavior.

An alternative possibility may be that children presenting to sleep disorders centers are more likely to exhibit PLMD, whether or not they are diagnosed with ADHD. Chervin and Hedger (35) found that $26 \%$ of children presenting to their sleep disorders center who underwent PSG had PLMI $\geq 5$ and found no association between PLMD and inattention/ hyperactivity in children. Conversely, Picchietti and Walters (18) discovered that $91 \%$ of children with PLMD in their clinic had a diagnosis of ADHD. The disparity in these findings is therefore difficult to reconcile, and further indicates the need for additional research on the prevalence and significance of PLMD in children with ADHD.

The association between sleep architecture abnormalities and ADHD remains unclear. A review of the published studies 
that used polysomnography for objective sleep assessment yields inconsistent results in regards to REM sleep characteristics, latency to sleep onset, and total sleep duration in children with ADHD (4). Our present findings indicate that preadolescent children with ADHD have significantly increased REM sleep latency with reduction in percentage of REM sleep compared with controls. These findings appear to be consistent with some of the previously reported data on REM sleep characteristics in ADHD children that revealed prolonged latency to the first REM sleep period and reduced amount of REM sleep $(20,23)$.

In one of the most recent studies, 30 preadolescent medication-free children with ADHD were compared with 19 agematched controls using PSG with video monitoring (36). In this latter study, the mean REM sleep latency reported in the ADHD sample $(157.8 \mathrm{~min})$ was remarkably similar to the mean REM sleep latencies of the clinical ADHD group (161.1 $\mathrm{min})$ and that of the community ADHD sample $(159.0 \mathrm{~min})$ in the present study. However, our control group of children exhibited much shorter REM sleep latency of $121.3 \mathrm{~min}$ compared with the $143.2 \mathrm{~min}$ reported by Konofal et al. (36). Because sleep characteristics are greatly influenced by age, the narrow age range and large sample sizes of both our controls $(n$ $=49)$ and ADHD subjects $(n=100)$ permitted us to uncover statistically significant differences in objective sleep measures, while eliminating many of the biases that may have affected previous studies.

One of the obvious limitations of our study was that PSG was conducted for only one night. First-night effect is one of the factors that may affect REM sleep latency, and the increased REM sleep latency among ADHD children could essentially underlie the fact that ADHD children are more sensitive to changes in the environment. However, when the sleep EEG dynamics were followed in ADHD children for four consecutive nights, there was a tendency to maintain prolonged REM sleep latency across all the nights (20).

Medications and psychiatric co-morbidities could affect REM sleep as well as sleep continuity measures. Even though there is no clear evidence for stimulant medication directly affecting REM sleep, a later onset to the first REM sleep period in patients treated with psychostimulants has been reported $(37,38)$. In a recent study, however, we found that stimulant medication had little effect on both subjective and objective sleep characteristics (39) in preadolescent children. Psychiatric co-morbidities such as anxiety, depression, and behavioral disorders may have a significant impact on sleep disturbances reported by parents (40). In this present study, we excluded children with co-morbid diagnoses and those treated with antidepressant and antipsychotic medications to avoid introduction of bias to the study.

Several methodological issues in the current study deserve further comment. First, none of the children included into the study was rigorously assessed using structured diagnostic interviews and rating scales to establish diagnosis of ADHD and determine whether additional psychiatric co-morbidities were present. However, we attempted to gain a more objective measure of hyperactivity by requiring that all of the ADHDcl had a professional diagnosis and assessing all of the ADHD- com group with a validated rating scale, such that selection of both ADHD children and controls was not based strictly on parental reports. A second limitation is that the sleep/wake schedules of our cohort were not controlled by actigraphic recordings before the PSG, and effects of partial sleep loss and inconsistent sleep schedules cannot be excluded as potential modifiers of sleep characteristics. A third limitation was, as mentioned above, the use of single-night PSG for assessment of sleep characteristics without having children acclimated to sleeping in an unfamiliar environment, therefore making it difficult to evaluate the role of adaptation processes, which may differ in ADHD children and normal controls. Finally, although we have recently developed normative data in children's sleep propensity (41), we did not assess for objective measures of daytime sleepiness, thereby restricting our ability to interpret increased parental reports of excessive daytime sleepiness (42) in clinical samples of ADHD compared with ADHDcom and controls.

\section{CONCLUSION}

Consistent with previous findings, we show that parental reports demonstrate an increased frequency of sleep problems among children with ADHD. We have further confirmed and expanded on the reduction in the proportion of REM sleep and prolongation of REM sleep latency in children with ADHD. However, increased PLMD with associated arousals was present only in the clinical sample of ADHD children, suggesting that this condition should not be extrapolated to all children with ADHD and could represent a unique subset of ADHD children with nocturnal motor hyperactivity.

Acknowledgments. The authors thank the parents and children who participated in this study for their cooperation.

\section{APPENDIX}

\section{1: The Sleep Questionnaire}

\section{Child's demographic information}

Name:

Address:

Telephone number:

Date of birth:

Gender:

Ethnicity:

\section{Father's demographic information}

Profession:

Highest level graduated from: junior high/high school/ college/graduate school

Snore: yes/no

Smoke: yes/no

Adenoids or tonsils removed: yes/no

Mother's demographic information

Profession:

Highest level graduated from: junior high/high school/ college/graduate school

Snore: yes/no

Smoke: yes/no 
Adenoids or tonsils removed: yes/no

Pregnancy: normal/abnormal

Pregnancy: full term/preterm

Child

Medical problems:

Medications:

Allergies:

Birth weight:

Adenoids or tonsils removed: yes/no

Vision problems: yes/no

Hearing problems: yes/no

Poor appetite: yes/no

Poor growth: yes/no

Ear infections: yes/no

Asthma: yes/no

Frequent colds and/or flus: yes/no

Constant runny nose: yes/no

Does child sleep alone, share with 1 , share with 2 , share with

3 , share with $>3$

Do any of his/her siblings snore? yes/no

Does your child have ADHD (also called hyperkinetic/ attention deficit)? yes/no

Is your child hyperactive? yes/no

Is your child on any ADHD medication? yes/no; which one:

How long does your child sleep at night?

At what time does your child go to bed?

At what time does your child wake up?

The following questions can be answered by "never," "rarely," "occasionally," frequently," or "almost always":

Have you seen or heard your child having nightmares that he/she does not remember the next day?

Has he/she expressed fear of sleeping in the dark?

Is your child easy to wake up in the morning?

Does your child go to bed willingly?

Is he/she a restless sleeper?

Have you seen your child smiling during sleep?

Does he/she wake up at night?

Have you heard your child talking during his/her sleep?

Have you observed him/her sleepwalking?

While asleep, does he/she ever sit up in bed?

Does he/she grind his/her teeth during sleep?

Have you heard your child laugh during sleep?

Has your child told you about having a frightening dream?

Have you observed repetitive actions such as rocking or

head banging during sleep?

Does he/she have problems with bed wetting?

Have you observed your child having a nightmare during which he/she appeared extremely afraid or terrified?

Have you looked in on your child and discovered he/she was crying while asleep?

Has he/she told you about having a pleasant dream?

Does your child complain about difficulties going to sleep?

Does your child get up to go to the bathroom during the night?

Does your child stop breathing during sleep?

Does your child struggle to breathe while asleep?

Does your child fall asleep easily?
Do you ever shake your child to make him/her breathe again when asleep?

Do your child's lips ever turn blue or purple while asleep? Are you ever concerned about your child's breathing during sleep?

How often does your child snore?

How loud is the snore? (the responses to this question are "mildly quiet," "medium loud," "loud," "very loud," and "extremely loud")

How often does your child have a sore throat?

Does your child complain of morning headaches?

Is your child a daytime mouth breather?

Is your child sleepy during the daytime?

Does your child fall asleep at school?

Does your child fall asleep while watching television?

\section{REFERENCES}

1. Kaplan BJ, McNichol J, Conte RA, Moghadam HK 1987 Sleep disturbance in preschool-aged and hyperactive and nonhyperactive children. Pediatrics 80:839-844

2. Ali NJ, Pitson D, Stradling JR 1993 Snoring, sleep disturbance and behaviour in 4-5 year olds. Arch Dis Child 68:360-366

3. Ball JD, Tiernan M, Janusz J, Furr A 1997 Sleep patterns among children with attention-deficit hyperactivity disorder: a re-examination of parental perceptions. J Pediatr Psychol 22:389-398

4. Corkum P, Tannock R, Moldofsky H 1998 Sleep disturbance in children with attention-deficit hyperactivity disorder. J Am Acad Child Adolesc Psychiatry 37:637646

5. Ring A, Stein D, Barak Y, Teicher A, Hadjez J, Elizur A, Weizman A 1998 Sleep disturbances in children with attention-deficit/hyperactivity disorder: a comparative study with healthy siblings. J Learn Disabil 31:572-578

6. Stein MA 1999 Unraveling sleep problems in treated and untreated children with ADHD. J Child Adolesc Psychopharmacol 9:157-168

7. Owens J, Maxim R, Nobile C, McGuinn M, Msall M 2000 Parental and self-report of sleep in children with attention deficit/hyperactivity disorder. Arch Pediatr Adolesc Med 154:549-555

8. Corkum P, Tannock R, Moldofsky H, Hogg-Johnson S, Humphries T 2001 Actigraphy and parental ratings of sleep in children with attention deficit/hyperactivity disorder (ADHD). Sleep 24:303-312

9. American Psychiatric Association 1980 Diagnostic and Statistical Manual of Mental Disorders, $3^{\text {rd }}$ Edition (DSM-III). American Psychiatric Association, Washington, DC

10. Guilleminault C, Eldridge F, Simmons FB, Dement WC 1976 Sleep apnea in eight children. Pediatrics 58:28-31

11. Brouillette RT, Fernbach SK, Hunt CE 1982 Obstructive sleep apnea in infants and children. J Pediatr 100:31-40

12. Stradling JR, Thomas G, Warley ARH, Williams P, Freeland A 1990 Effect of adenotonsillectomy on nocturnal hypoxaemia, sleep disturbance, and symptoms in snoring children. Lancet 335:249-253

13. Ali NJ, Pitson D, Stradling JR 1996 Sleep disordered breathing: effects of adenotonsillectomy on behaviour and psychological functioning. Eur J Pediatr 155:56-62

14. Chervin RD, Dillon JE, Bassetti C, Ganoczy DA, Pituch KJ 1997 Symptoms of sleep disorders, inattention, and hyperactivity in children. Sleep 20:1185-1192

15. Guilleminault C, Pelayo R 1998 Narcolepsy in prepubertal children. Ann Neurol 43:135-142

16. Picchietti DL, Walters AS 1996 Restless legs syndrome and periodic limb movement disorder in children and adolescents: comorbidity with attention-deficit hyperactivity disorder. Child Adolesc Psychiatr Clin North Am 5:729-740

17. Picchietti DL, England SJ, Walters AS, Willis K, Verrico T 1998 Periodic limb movement disorder and restless legs syndrome in children with attention-deficithyperactivity disorder. J Child Neurol 13:588-594

18. Picchietti DL, Walters AS 1999 Moderate to severe periodic limb movement disorder in childhood and adolescence. Sleep 22:297-300

19. Picchietti DL, Underwood DJ, Farris WA, Walters AS, Shah MM, Dahl RE, Trubnick LJ, Bertocci MA, Wagner M, Hening WA 1999 Further studies on periodic limb movement disorder and restless legs syndrome in children with attention-deficithyperactivity disorder. Mov Disord 14:1000-1007

20. Busby K, Firestone P, Pivik RT 1981 Sleep patterns in hyperkinetic and normal children. Sleep 4:366-383

21. Greenhill L, Puig-Antich J, Goetz R, Hanlon C, Davies M 1983 Sleep architecture and REM sleep measures in prepubertal children with attention deficit disorder with hyperactivity. Sleep 6:91-101

22. Busby K, Pivik RT 1985 Auditory arousal thresholds during sleep in hyperkinetic children. Sleep 8:322-341 
23. Ramos Platon MJ, Vela Bueno A, Espinar Sierra J, Kales S 1990 Hypnopolygraphic alterations in attention deficit disorder (ADD) children. Int J Neurosci 53:87-101

24. Kahn A 1982 Sleep REM latency in hyperkinetic children. Am J Psychiatry 139:1359-1364

25. Chervin RD, Archbold KH, Dillon JE, Panahi P, Pituch KJ, Dahl RE, Guilleminaul C 2002 Inattention, hyperactivity, and symptoms of sleep-disordered breathing. Pediatrics 109:449-456

26. Chervin RD, Archbold KH 2001 Hyperactivity and polysomnographic findings in children evaluated for sleep-disordered breathing. Sleep 24:313-320

27. Conners CK 1989 Conners' Parent Rating Scales. MHS Publishing, New York

28. Walters S, Mandelbaum DE, Lewin DS, Kugler S, England SJ, Miller M 2000 Dopaminergic therapy in children with restless legs/periodic limb movements in sleep and ADHD. Pediatr Neurol 22:182-186

29. Gozal D 1998 Sleep-disordered breathing and school performance in children Pediatrics 102:616-620

30. Rechtschaffen A, Kales A 1968 A Manual of Standardized Terminology, Techniques and Scoring Systems for Sleep Stages of Human Subject. U.S. Government Printing Office, U.S. Public Health Service, Washington DC

31. Anonymous 1992 EEG arousals: scoring rules and examples: a preliminary report from the Sleep Disorders Atlas Task Force of the American Sleep Disorders Association. Sleep 15:173-184

32. Anonymous 1996 Standards and indications for cardiopulmonary sleep studies in children. American Thoracic Society. Am J Resp Crit Care Med 153:866-878

33. Marcus CL, Omlin KJ, Basinski DJ, Bailey SL, Rachel AB, Keens TG, Davidson Ward SL 1992 Normal polysomnographic values for children and adolescents. Am Rev Respir Dis 156:1235-1239
34. Diagnostic Classification Steering Committee 1990 Periodic limb movement disorder In: International Classification of Sleep Disorders: Diagnostic and Coding Manual. American Sleep Disorders Association, Rochester, MN, pp 69-71

35. Chervin RD, Hedger KM 2001 Clinical prediction of periodic leg movements during sleep in children. Sleep Med 2:501-510

36. Konofal E, Lecendreux M, Bouvard M, Mouren-Simeoni M 2001 High levels of nocturnal activity in children with attention-deficit hyperactivity disorder: a video analysis. Psychiatry Clin Neurosci 55:97-103

37. Feinberg I, Hibi S, Braun M, Cavness C, Westerman G, Small A 1974 Sleep amphetamine effects in MBDS and normal subjects. Arch Gen Psychiatry 31:723731

38. Small A, Hibi S, Feinberg I 1971 Effects of dextroamphetamine sulfate on EEG sleep patterns of hyperactive children. Arch Gen Psychiatry 25:369-380

39. O'Brien LM, Ivanenko A, Crabtree VM, Holbrook CR, Bruner JL, Klaus CJ, Gozal D 2003 The effect of stimulants on sleep chracteristics in children with attention deficit/hyperactivity disorder. Sleep Med, in press

40. Mick E, Bierderman J, Jetton J, and Faraone S 2000 Sleep disturbances associated with attention deficit hyperactivity disorder: the impact of psychiatric comorbidity and pharmacotherapy. J Child Adolesc Psychopharmacol 10:223231

41. Gozal D, Wang M, Pope DW 2001 Objective sleepiness measures in pediatric obstructive sleep apnea. Pediatrics 108:693-697

42. Lecendreux M, Konofal E, Bouvard M, Falissard B, Mouren-Simenoi MC 2000 Sleep and alertness in children with ADHD. J Child Psychol Psychiatry 41:803812 